\title{
BMJ Open Diet quality during preconception or pregnancy and gestational weight gain: protocol for a systematic review and meta-analysis
}

\author{
Yamei Yu (D) , ${ }^{1}$ Isabelle Hardy (D) , ${ }^{2}$ Wenguang Sun, ${ }^{3}$ Dean A Fergusson, ${ }^{1,4}$ \\ William Fraser, ${ }^{2,5}$ Lise Dubois $^{1}$
}

To cite: Yu Y, Hardy I, Sun W, et al. Diet quality during preconception or pregnancy and gestational weight gain: protocol for a systematic review and meta-analysis. BMJ Open 2020;10:e033130. doi:10.1136/ bmjopen-2019-033130

- Prepublication history and additional material for this paper are available online. To view these files, please visit the journal online (http://dx.doi. org/10.1136/bmjopen-2019033130).

Received 23 July 2019 Revised 11 January 2020 Accepted 16 January 2020

Check for updates

(c) Author(s) (or their employer(s)) 2020. Re-use permitted under CC BY-NC. No commercial re-use. See rights and permissions. Published by BMJ.

For numbered affiliations see end of article.

Correspondence to

Dr Lise Dubois;

Idubois@uottawa.ca

\section{ABSTRACT}

Introduction Inappropriate gestational weight gain (GWG), including inadequate and excessive GWG, has become pandemic across nations and continents. This review aims to synthesise the evidence on the correlation between diet quality and GWG. If this association is confirmed, improving diet quality could become an intervention target in the efforts to reduce inappropriate GWG.

Methods and analysis We will conduct a systematic review of all prospective cohort studies on diet quality in preconception or pregnancy and GWG. Our secondary outcomes include gestational diabetes, pre-eclampsia and birth weight. A comprehensive search of all published articles in MEDLINE ALL (Ovid), Embase (Ovid), Food Science and Technology Abstracts (Ovid) and CINAHL (EBSCOHost), from database creation to 20 April 2019, will be conducted. Studies will be screened for eligibility by title, abstract and full text in duplicate by two independent reviewers. Study quality and risk of bias will be assessed using the adapted Newcastle-Ottawa Scale. Results will be reported following the meta-analysis of observational studies in epidemiology guidelines. If sufficient data are available, a meta-analysis will be conducted to synthesise the effect size reported as $\mathrm{OR}$ with $95 \% \mathrm{Cl}$ using both fixed-effect and random-effect models. $\left.\right|^{2}$ statistics and visual inspection of the forest plots will be used to assess heterogeneity and identify the potential sources of heterogeneity. Publication bias will be assessed by visual inspections of funnel plots and Egger's test.

Ethics and dissemination Formal ethical approval is not required as no primary data will be collected. We aim to publish the results of this study in a peer-reviewed journal and present them at conferences and scientific meetings to promote knowledge transfer.

PROSPERO registration number CRD42019128732

\section{INTRODUCTION}

Defining diet quality

Diet quality is a relatively new concept measured by scoring diet in terms of ' $a$ priori' defined adherence to dietary guidelines or a specific pattern. Compared with single nutrient or single food group measures, diet quality enables research on overall diet using
Strengths and limitations of this study

- Compared with diet quantity, or single nutrients/food groups, the importance of overall diet quality has not been evaluated in correlation with gestational weight gain and subsequent maternal and childhood outcomes. This study is the first to synthesise the evidence regarding the association between diet quality and gestational weight gain.

- We will only include prospective cohort studies to avoid recall bias in dietary assessment and reverse causation in case-control and cross-sectional studies.

- The main methodological limitations of this systematic review are the exclusion of abstracts and foreign-language publications.

- Another potential limitation we anticipate is that we might encounter different definitions of diet quality and the secondary outcomes, which will limit our ability to pool the results in meta-analysis.

broader components of food groups, based on the best available knowledge concerning associations between diet and health. Diet quality indices also differentiate from a data-driven dietary pattern analysis which is studied ' $a$ posteriori using factor or cluster analysis. ${ }^{1}$ Some diet quality indices are based on national dietary guidelines, such as the Healthy Eating Index $(\mathrm{HEI})^{23}$ in USA, which was developed in 2008 to assess the alignment of diet to Dietary Guidelines for Americans. Newer versions (HEI$2010^{4}$ and HEI-2015 ${ }^{5}$ ) correspond to evolving versions of the dietary guidelines. Other diet quality indices evaluate adherence to certain healthy dietary patterns such as Mediterraneanstyle patterns ${ }^{6}$ or dietary approaches to stop hypertension-style patterns. ${ }^{7}$ Systematic reviews have identified a list of diet quality indices including Diet Quality Index, Dietary Guideline Index, Dietary Diversity Score, Recommended Food Score and so on. ${ }^{89}$ 


\section{Defining gestational weight gain (GWG) and its importance}

Appropriate weight gain during pregnancy is important for maternal and infant health. In $2009,{ }^{10}$ the Institute of Medicine (IOM; now known as the National Academy of Medicine) updated the GWG guidelines ${ }^{11}$ to provide specific recommendations regarding the ideal GWG. The new guideline incorporated WHO categories of maternal body mass index (BMI) and recommended less GWG for overweight and obese women. Briefly, the IOM recommended $12.5-18 \mathrm{~kg}$ of total GWG for singleton pregnancies with a prepregnancy BMI categorised as underweight $(\mathrm{BMI}<18.5), 11.5-16 \mathrm{~kg}$ for normal weight (BMI 18.5-24.9), 7-11.5 kg for overweight (BMI 25-29.9) and $5-9 \mathrm{~kg}$ for obese $(\mathrm{BMI} \geq 30)$. Total GWG within the IOM recommendations will be considered as appropriate GWG, above the recommendations will be considered as excessive GWG and below the recommendations will be considered as inadequate GWG.

Inappropriate GWG has a significant effect on perinatal outcomes, independent of maternal BMI. A systematic review and meta-analysis ${ }^{12}$ found that excessive GWG is associated with an increased risk of large for gestational age (OR $1.85,95 \%$ CI 1.76 to 1.95), macrosomia (1.95, 1.79 to 2.11$)$ and caesarean delivery $(1.30,1.25$ to 1.35$)$. Low GWG is related to increased risk of small for gestational age $(1.53,1.44$ to 1.64$)$ and preterm birth (1.70, 1.32 to 2.20 ). Higher maternal GWG is also associated with higher risks of gestational hypertensive disorders, pre-eclampsia, preterm birth and gestational diabetes. ${ }^{13}$ Although overweight and obese women present high rates of both excessive GWG, and gestational diabetes and pre-eclampsia, GWG has not been consistently found to mediate these complications. ${ }^{12} 14$

Inappropriate GWG also has long-term effects on maternal and offspring health. In a meta-analysis, ${ }^{15}$ women with a GWG above recommended levels retained, on average, an additional $3.06 \mathrm{~kg}$ (95\% CI 1.50 to 4.63 ) after 3 years, and $4.72 \mathrm{~kg}$ (2.94 to 6.50$)$ after $\geq 15$ years post partum, compared with women with GWG within the recommendations. In non-overweight women, excessive GWG has been found to triple the risk of becoming overweight after pregnancy $(3.19,1.87$ to 5.44$) .{ }^{16}$ In the offspring, excessive maternal GWG has been associated with increased adiposity ${ }^{17}$ and an increased risk of overweight/obesity ${ }^{18}$ during childhood.

Considering the global epidemic of inappropriate GWG, with prevalence of excessive and inadequate GWG, respectively, reaching $50 \%$ and $20 \%$ across continents and ethnicities, ${ }^{19}$ it is urgent that effective interventions be put in place.

\section{Association between diet quality and GWG}

Pregnancy is a critical period when energy and nutrient intake must support growth of maternal and fetal tissues. Low diet quality characterised by high saturated fat, refined grains, free sugars, low fibre intake may contribute to excessive energy intake which may be a risk factor for excessive GWG and inadequate vital nutrient intake. Thus, adjusting diet quality could be a target for nutritional intervention to reduce excessive weight gain.

Although dietary patterns are difficult to change, pregnancy appears as a window of opportunity where women are willing to adopt healthier dietary habits. ${ }^{20}$ Thus, pregnancy is a critical intervention period to implement dietary interventions targeting both women and their families. Considering that the offspring share similar dietary patterns with the parents, a good diet pattern can be transmitted to the next generation with potential longlasting effects on health.

\section{Why is it important to do this review?}

With the recent development of prospective birth cohorts, evidence of an association between diet quality and GWG is accumulating. However, no systematic review has evaluated this association. One systematic review evaluated the relationship between macronutrients intake and $\mathrm{GWG},{ }^{21}$ but none has reviewed the evidence on the relationship between global diet quality and GWG. Consequently, diet quality has been neglected as a risk factor and potential intervention target for inappropriate GWG. We would like to fill this knowledge gap by providing a robust evidence synthesis on the subject. This work will guide effective interventions and policy making to prevent inappropriate GWG, and its short-term and long-term consequences.

\section{Research objectives}

The objective of our study is to systematically review prospective cohort studies that explore the association between diet quality and GWG. Our secondary aim will be to explore whether this association is mediated by energy intake, and whether diet quality has further impact on clinical outcomes including gestational diabetes, preeclampsia and birth weight. If significant heterogeneity is identified, we will conduct subgroup analyses to explore potential factors that modify the association between diet quality and GWG. Potential effect modifying factors include the origin of the study population (developed/ developing countries, urban/rural areas), the characteristics of the population (overweight or obese/general pregnant women) and the timing of dietary evaluation (preconception/trimester of pregnancy).

\section{METHODS AND ANALYSIS}

\section{Patient and public involvement}

Patients and the public were not involved in the design and conception of this study.

\section{Eligibility criteria}

Studies will be screened and selected according to the criteria specified below.

\section{Study designs}

Only prospective cohort studies of human subjects will be included in this systematic review. Case-control studies will be excluded because they are retrospective and may lead to recall bias in dietary assessment. ${ }^{22}$ Secondary 
analysis of the control arm of randomised control trials will be included, since this design mimics a prospective cohort and excludes the effect of the intervention. In order to avoid recall bias in dietary assessment and avoid reverse causation, we will only include studies where dietary assessment is completed before the outcome measurement. We will exclude other study types such as narrative and systematic reviews, experimental or quasiexperimental trials, case-control studies, cross-sectional studies, case series and case reports.

\section{Publication type}

Only full-text articles published in scholarly peer-reviewed journals in English will be included. Only articles published in English will be included. We will exclude abstracts, unpublished grey literature, commentaries, letters, reviews and editorials, meeting proceedings, theses and dissertations, books, treatment guidelines or manuals. ${ }^{23}$

\section{Participants}

We are interested in studies of women from the general population in the preconception period or pregnancy. Studies including only women with a specific disease, such as heart disease, diabetes, hypertension or gestational diabetes, will be excluded because these populations may already adhere to specifically prescribed dietary patterns, which might lead to reverse causation in the association between diet and the outcomes. Studies of diet quality in the general population including some women with a specific disease will be included. Studies of women with overweight or obesity will also be included. Most included women are expected to be 18-45years at the time of study participation, but general population studies including some minor or older participants will be included. We will exclude studies focusing only on participants $<18$ years old such as studies of teenagers.

\section{Exposure}

We are interested in the difference in the outcomes of women exposed to high diet quality, compared with women exposed to low diet quality in the same population. Diet quality will be assessed systematically using prespecified scoring scales and validated dietary assessment methods including food diaries, food recalls or food frequency questionnaires. Diet quality analysed as both categorical and continuous variables will be included. Studies with exposures defined as a single or few nutrients or food groups will be excluded.

\section{Outcomes}

Our primary outcome is GWG according to the IOM recommendations, and our secondary outcomes include gestational diabetes, pre-eclampsia, preterm delivery, delivery by caesarean section and birth weight for gestational age. We will not exclude studies based on the definition of the outcomes because we anticipate that there will be discrepancies in the definition of outcomes in the target studies. We will extract outcomes in all data forms (eg, dichotomous, continuous) as reported in the included studies. The definition of the outcomes in each study will be recorded.

\section{Information sources}

Four databases will be searched: MEDLINE ALL (Ovid), Embase (Ovid), Food Science and Technology Abstracts (Ovid) and CINAHL (EBSCOHost). All databases will be searched from the date of database creation to the cut-off date of 20 April 2019. In order to further ensure a comprehensive literature search, reference lists of included studies and relevant review articles identified through the search will be checked for additional references. Authors of relevant published abstracts will be contacted to verify if full-text manuscripts have been published. Finally, we will circulate a bibliography of the included articles to the systematic review team for feedback.

\section{Search strategy}

Both medical subject headings and text keywords were used to develop the search strategies. Two groups of keywords were used: 'preconception or pregnancy', and diet quality. The Medline search strategy was developed with inputs from the whole research team and the health science librarian (LS) with expertise in systematic review research strategies. After the Medline search strategy was finalised, it was adapted to the syntax of other databases. The search terms were then peer reviewed by a second health science librarian not otherwise associated with the project. ${ }^{24}$ The detailed search terms used in OVID Medline are shown in online supplementary file 1.

\section{Study selection}

The publications identified with the search process will be uploaded to the Covidence software, which is an online service working in partnership with Cochrane to improve the production and use of systematic reviews for health and well-being. Two review authors ( $\mathrm{YY}$ and $\mathrm{IH}$ ) will independently screen the titles and abstracts of the search results to exclude articles that do not meet the eligibility criteria. All articles that seem to meet the inclusion criteria in the title and abstract screening or those with uncertainties will be assessed with full text. Reasons for exclusion in the fulltext screening will be recorded. Any disagreement arising during the selection process will be resolved by discussion with a third reviewer. The process of study selection will be reported using the Preferred Reporting Items for Systematic Reviews and Meta-Analyses flow diagram. ${ }^{25}$

\section{Data collection process}

YY and IH will independently extract all information related to the research question from the included studies for both narrative synthesis and potential meta-analysis. A predefined extraction sheet will be used. We will also contact the authors if related data are ambiguous or not included in the publications. Any disagreement arising during the extraction process will be resolved by discussion with a third reviewer LD.

The following data will be extracted from all studies meeting the inclusion criteria: 
1. Study authors.

2. Year of study.

3. Country/countries where study was conducted.

4. Population characteristics (including their ethnicity, age, parity and so on) with inclusion/exclusion criteria.

5. Study design and characteristics.

6. Number of participants.

7. Exposure information on diet quality, including diet assessment method, definition of diet quality, calculation, time of measurement (preconception or gestational age).

8. Outcomes: definition and time of measurement.

9. Conflicts of interest and funding.

10. Statistical analysis.

11. Confounders adjusted for in the statistical analysis.

12. Selected main findings.

\section{Risk of bias in individual studies}

YY and IH will independently assess the quality and risk of bias of the included studies in duplicates. Discrepancies between the two reviewers will be resolved by discussion. If necessary, a third author of the team will be consulted to achieve consensus. The quality of the studies will be assessed with the Newcastle-Ottawa Scale. ${ }^{26}$ In this scale, a maximum of 9 points can be awarded to each cohort study: 4 for selection, which evaluates selection bias ascertainment of exposure; 2 for comparability, which requires control of appropriate confounders and 3 for the outcome, which requires a high quality measurement of the outcomes and follow-up. The sum of points for all subscale items will be used to categorise overall study quality as either high $(>7)$, moderate $(5-7)$ or low $(<5)$ to decide on the likelihood of reliability of the outcome reports. Justification from the study report will be supplied to support the judgement as appropriate.

\section{Data synthesis}

Risk ratios with $95 \%$ CI will be used to summarise the association between diet quality (the highest vs lowest levels of diet quality scores) and GWG (excessive or insufficient GWG compared with appropriate GWG category according to IOM guideline) and the association between diet quality and other categorical outcomes. Weighted mean differences with 95\% CI will be used for continuous outcomes. We are anticipating different scales of reporting diet quality across studies, including 1 absolute unit increase, 1 SD increase, tertiles, quartiles, quintiles. The different scales will be transformed to calculate the effect size in the top tertile of diet quality scores compared with the bottom tertile using the method reported in previous studies. ${ }^{27-29}$ The homogeneity of the study results will be assessed using $\mathrm{I}^{2}$ statistic and by visual inspection of the forest plots. The rough guide for interpretation of $\mathrm{I}^{2}$ are as follows: $0 \%-40 \%$ may present low heterogeneity, $30 \%-60 \%$ may present moderate heterogeneity, 50\%-90\% may represent substantial heterogeneity and $75 \%-100 \%$ is considerable heterogeneity. ${ }^{30}$ If there is a substantial amount of heterogeneity $(\geq 75 \%)$, the sources of heterogeneity will be examined through subgroup analyses examining factors including origin of the study population (developed/developing countries, urban/rural areas), the characteristics of the population (overweight or obese/general pregnant women) and the timing of dietary evaluation (preconception/trimesters of pregnancy). Considering the possibility of different covariates used in each study, we are planning to do subgroup analysis separating studies including or not including the important covariates including prepregnancy BMI, socioeconomic background, parity and so on, if a sufficient number of studies are available. If appropriate, we will also make exclusions based on the sensitivity analysis excluding studies with high risk of bias to improve the homogeneity of the results. A quantitative analysis (metaanalysis) will be done using Review Manager (RevMan) V.5.3 (The Nordic Cochrane Centre, The Cochrane Collaboration, 2014). Populational characteristics and exposure/ outcome definitions may vary between the target observational studies, so pooling data using a random-effect model may seem more reasonable. But presenting results from both models in a sensitivity analysis may be informative. ${ }^{31}$ In this study, both fixed-effect model and random-effect model will be used to estimate the summary statistics and 95\% CIs. If there is a sufficient number of studies on the mediation effect of total energy on the association between diet quality and GWG, we will use correlation-based or parameter-based approaches, as appropriate, to conduct a meta-analytic structural equation modelling as reviewed by Cheung and Cheung. ${ }^{32}$ If the included studies are not sufficiently homogenous to conduct a meta-analysis or if three or fewer studies use similar measuring methods, a narrative synthesis will be provided to report relevant findings from the included studies.

\section{Publication bias and small study effects}

Publication bias will be assessed in all analyses synthesising 10 or more studies to ensure adequate power in the analysis. ${ }^{30}$ For investigation of the effect of small studies and publication bias, data from included studies will be entered into a funnel plot asymmetry test if we have at least 10 studies in the meta-analysis. Egger's statistical test will be implemented using STATA/SE V.13 (Stata Corp).

\section{Confidence in cumulative evidence}

The quality of supporting evidence will be assessed by the Grades of Recommendation, Assessment, Development and Evaluation. ${ }^{33}$

\section{Amendments}

This is an original research protocol, as opposed to an amendment of a previously completed protocol. If the protocol requires major amendments, the changes will be documented and updated via PROSPERO and stated in the final review manuscript.

Author affiliations

${ }^{1}$ School of Epidemiology and Public Health, Faculty of Medicine, University of Ottawa, Ottawa, Ontario, Canada 
${ }^{2}$ Department of Obstetrics and Gynecology, Faculty of Medicine and Health Sciences, Université de Sherbrooke, Sherbrooke, Quebec, Canada

${ }^{3}$ The International Peace Maternity and Child Health Hospital, School of Medicine,

Shanghai Jiao Tong University, Shanghai, China

${ }^{4}$ Clinical Epidemiology Program, Ottawa Hospital Research Institute, Ottawa, Ontario, Canada

${ }^{5}$ Centre de Recherche du Centre hospitalier Universitaire de Sherbrooke (CRCHUS), Sherbrooke, Ottawa, Canada

Acknowledgements The authors would like to thank Lindsey Sikora (librarian) for counselling in developing the searching strategies. We also thank Dr Jean-Patrice Baillargeon for critical reviews and suggestions in formulating the systematic review protocol.

Collaborators Lindsey Sikora; Jean-Patrice Baillargeon.

Contributions YY is the guarantor of the review. YY, WS, WF and LD contributed to the conception of the research question. YY, IH, WS and DAF contributed to the development of search strategies, eligibility criteria and methodology for data synthesis. YY, IH, WS, LD, WF and DAF contributed to drafting of the protocol and provided approval for the version submitted for publishing. YY and IH will work in duplicate to screen the titles and abstracts of all the materials obtained using the search strategy to exclude the articles that do not meet the eligibility criteria. YY and $\mathrm{IH}$ will evaluate the potentially eligible studies with the full text and further exclude studies with documentation of the reason for exclusion. All authors will contribute to the bias assessment strategy and data extraction criteria. YY and IH will independently extract data from the included studies using a predefined extraction sheet. YY and IH will analyse the data and draft the results. All authors will read, provide feedback and approve the final manuscript.

Funding This research project is sponsored jointly by the Canadian Institutes of Health Research (CIHR) (Grant HLT 151517) and the National Natural Sciences Foundation of China (NSFC) (Grant No. 81661128010).

Competing interests None declared.

Patient consent for publication Not required.

Ethics approval Formal ethical approval is not required as no primary data will be collected. We aim to publish the results of this study in a peer-reviewed journal and present them at conferences and scientific meetings to promote knowledge transfer.

Provenance and peer review Not commissioned; externally peer reviewed.

Open access This is an open access article distributed in accordance with the Creative Commons Attribution Non Commercial (CC BY-NC 4.0) license, which permits others to distribute, remix, adapt, build upon this work non-commercially, and license their derivative works on different terms, provided the original work is properly cited, appropriate credit is given, any changes made indicated, and the use is non-commercial. See: http://creativecommons.org/licenses/by-nc/4.0/.

\section{ORCID iDs}

Yamei Yu http://orcid.org/0000-0002-5151-3397

Isabelle Hardy http://orcid.org/0000-0001-9996-2995

\section{REFERENCES}

$1 \mathrm{Hu}$ FB. Dietary pattern analysis: a new direction in nutritional epidemiology. Curr Opin Lipidol 2002;13:3-9.

2 Guenther PM, Reedy J, Krebs-Smith SM. Development of the healthy eating Index-2005. J Am Diet Assoc 2008;108:1896-901.

3 Guenther PM, Reedy J, Krebs-Smith SM, et al. Evaluation of the healthy eating Index-2005. J Am Diet Assoc 2008;108:1854-64.

4 Guenther PM, Casavale KO, Reedy J, et al. Update of the healthy eating index: HEl-2010. J Acad Nutr Diet 2013;113:569-80.

5 Krebs-Smith SM, Pannucci TE, Subar AF, et al. Update of the healthy eating index: HEl-2015. J Acad Nutr Diet 2018;118:1591-602.

6 Trichopoulou A, Costacou T, Bamia C, et al. Adherence to a Mediterranean diet and survival in a Greek population. N Engl J Med 2003;348:2599-608.

7 Fung TT, Chiuve SE, McCullough ML, et al. Adherence to a DASHstyle diet and risk of coronary heart disease and stroke in women. Arch Intern Med 2008;168:713-20.

8 Asghari G, Mirmiran P, Yuzbashian E, et al. A systematic review of diet quality indices in relation to obesity. Br J Nutr 2017;117:1055-65

9 Wirt A, Collins CE. Diet quality--what is it and does it matter? Public Health Nutr 2009;12:2473-92.
10 Rasmussen KM, Yaktine AL, eds. Weight gain during pregnancy: reexamining the guidelines. Washington, DC: National Academies Press, 2009.

11 Institute of Medicine (US) Committee on Nutritional Status During Pregnancy and Lactation. Nutrition during pregnancy: Part I weight gain: Part II nutrient supplements. Washington, DC: National Academies Press (US), 1990.

12 Goldstein RF, Abell SK, Ranasinha S, et al. Association of gestational weight gain with maternal and infant outcomes: a systematic review and meta-analysis. JAMA 2017;317:2207-25.

13 Santos S, Voerman E, Amiano P, et al. Impact of maternal body mass index and gestational weight gain on pregnancy complications: an individual participant data meta-analysis of European, North American and Australian cohorts. BJOG 2019;126:984-95.

14 Nohr EA, Vaeth M, Baker JL, et al. Combined associations of prepregnancy body mass index and gestational weight gain with the outcome of pregnancy. Am J Clin Nutr 2008;87:1750-9.

15 Nehring I, Schmoll S, Beyerlein A, et al. Gestational weight gain and long-term postpartum weight retention: a meta-analysis. Am J Clin Nutr 2011;94:1225-31.

16 Gunderson EP, Abrams B, Selvin S. The relative importance of gestational gain and maternal characteristics associated with the risk of becoming overweight after pregnancy. Int $J$ Obes Relat Metab Disord 2000;24:1660-8.

17 Hochner H, Friedlander Y, Calderon-Margalit R, et al. Associations of maternal prepregnancy body mass index and gestational weight gain with adult offspring cardiometabolic risk factors: the Jerusalem perinatal family follow-up study. Circulation 2012;125:1381-9.

18 Voerman E, Santos S, Patro Golab B, et al. Maternal body mass index, gestational weight gain, and the risk of overweight and obesity across childhood: an individual participant data meta-analysis. PLoS Med 2019;16:e1002744.

19 Goldstein RF, Abell SK, Ranasinha S, et al. Gestational weight gain across continents and ethnicity: systematic review and meta-analysis of maternal and infant outcomes in more than one million women. BMC Med 2018;16:153.

20 Vanstone M, Kandasamy S, Giacomini M, et al. Pregnant women's perceptions of gestational weight gain: a systematic review and meta-synthesis of qualitative research. Matern Child Nutr 2017;13.

21 Tielemans MJ, Garcia AH, Peralta Santos A, et al. Macronutrient composition and gestational weight gain: a systematic review. Am J Clin Nutr 2016;103:83-99.

22 Song JW, Chung KC. Observational studies: cohort and case-contro studies. Plast Reconstr Surg 2010;126:2234-42.

23 Fergusson D, Laupacis A, Salmi LR, et al. What should be included in meta-analyses? an exploration of methodological issues using the ISPOT meta-analyses. Int J Technol Assess Health Care 2000;16:1109-19.

24 McGowan J, Sampson M, Salzwedel DM, et al. PRESS Peer Review of Electronic Search Strategies: 2015 Guideline Statement. J Clin Epidemiol 2016;75:40-6.

25 Moher D, Liberati A, Tetzlaff J, et al. Preferred reporting items for systematic reviews and meta-analyses: the PRISMA statement. BMJ 2009;339:b2535.

26 Wells GA, Shea B, O'Connell D, et al. The Newcastle-Ottawa scale (NOS) for assessing the quality of nonrandomised studies in metaanalyses, 2011. Available: http://www.ohri.ca/programs/clinical_ epidemiology/oxford.asp

27 Danesh J, Collins R, Appleby P, et al. Association of fibrinogen, C-reactive protein, albumin, or leukocyte count with coronary heart disease: meta-analyses of prospective studies. JAMA 1998;279:1477-82.

28 Araujo AB, Dixon JM, Suarez EA, et al. Clinical review: endogenous testosterone and mortality in men: a systematic review and metaanalysis. J Clin Endocrinol Metab 2011;96:3007-19.

29 Chia A-R, Chen L-W, Lai JS, et al. Maternal dietary patterns and birth outcomes: a systematic review and meta-analysis. Adv Nutr 2019;10:685-95.

30 Higgins J, Green S, eds. Cochrane handbook for systematic reviews of interventions version 5.1.0. The Cochrane collaboration, 2011.

31 Dekkers OM, Vandenbroucke JP, Cevallos M, et al. COSMOS-E: guidance on conducting systematic reviews and meta-analyses of observational studies of etiology. PLoS Med 2019;16:e1002742.

32 Cheung MW-L, Cheung SF. Random-effects models for metaanalytic structural equation modeling: review, issues, and illustrations. Res Synth Methods 2016;7:140-55.

33 Guyatt GH, Oxman AD, Vist GE, et al. Grade: an emerging consensus on rating quality of evidence and strength of recommendations. $B M J$ 2008;336:924-6. 